\title{
Lamb-dip spectrum of methylacetylene and methyldiacetylene: precise rotational transition frequencies and parameters of the main isotopic species
}

\author{
G. Cazzoli and C. Puzzarini
}

Dipartimento di Chimica “G. Ciamician”, Università di Bologna, via Selmi 2, 40126 Bologna, Italy

e-mail: [gabriele.cazzoli; cristina.puzzarini]@unibo.it

Received 9 April 2008 / Accepted 18 May 2008

\section{ABSTRACT}

\begin{abstract}
Context. Methylacetylene and methyldiacetylene are the first members of the methylpolyynes series, $\mathrm{CH}_{3} \mathrm{C}_{2 n} \mathrm{H}(n=1,2)$. Their astrophysical relevance has motivated this spectroscopic study.

Aims. The aim of this investigation is to provide very accurate rest frequencies, as well as to improve the spectroscopic parameters available in the literature for these molecules.

Methods. The Lamb-dip technique was exploited in order to record the rotational spectra of $\mathrm{CH}_{3} \mathrm{CCH}$ and $\mathrm{CH}_{3} \mathrm{CCCCH}$ at subDoppler resolution in the millimeter- and submillimeter-wave frequency ranges. In addition, for $\mathrm{CH}_{3} \mathrm{CCH}$ rotational transitions in the THz region were recorded at Doppler resolution.

Results. We report the most accurate ground-state rotational parameters known at the moment for the main isotopic species of methylacetylene, as well as of methyldiacetylene.

Conclusions. Since both molecules are known to be of astrophysical relevance, we are confident that the improvement in the accuracy of the ground-state rotational parameters as well as of the rest frequencies of rotational transitions could be useful for future observations purposes.
\end{abstract}

Key words. techniques: spectroscopic - molecular data - methods: laboratory - ISM: molecules - radio lines: ISM

\section{Introduction}

Acetylenic chains, cyanopolyynes, and methylpolyynes are known to be molecules of astrophysical interest as a great deal of them has been detected in the interstellar medium. In particular, symmetric-top molecules are ideal probes for determining the kinetic temperature of the interstellar medium (ISM) because of the $K$-structure of the rotational transitions, which provides the quite unique opportunity of observing closely spaced lines with different excitation energies (i.e., temperature) simultaneously (Solomon et al. 1971, 1973).

Among methylpolyynes, $\mathrm{CH}_{3} \mathrm{C}_{2 n} \mathrm{H}(n=1,2, \ldots)$, methylacetylene and methyldiacetylene are the first $(n=1)$ and second $(n=2)$ members of the series, respectively, and they have been widely observed in the ISM. Concerning the former, its first detection in the ISM was reported by Snyder \& Buhl (1973) towards the galactic center source Sgr B2. Moreover, it was observed in extragalactic sources by Mauersberger et al. (1991). Since then, other observations took place (see, for example, Pratap et al. 1997; Agundez et al. 2008). Methyldiacetylene was first observed in the Taurus molecular cloud (TMC-1) in 1984 (MacLeod et al. 1984; Walmsley et al. 1984; Loren et al. 1984). After its first detection, more observations were reported in the literature (see, for example, Cernicharo et al. 2001). Furthermore, the recent detection of interstellar $\mathrm{CH}_{3} \mathrm{C}_{6} \mathrm{H}$ toward the TMC-1 cloud (Remijan et al. 2006) deserves to be mentioned.

In response to the need for more precise laboratory rest frequencies and, therefore, to improve the spectroscopic data

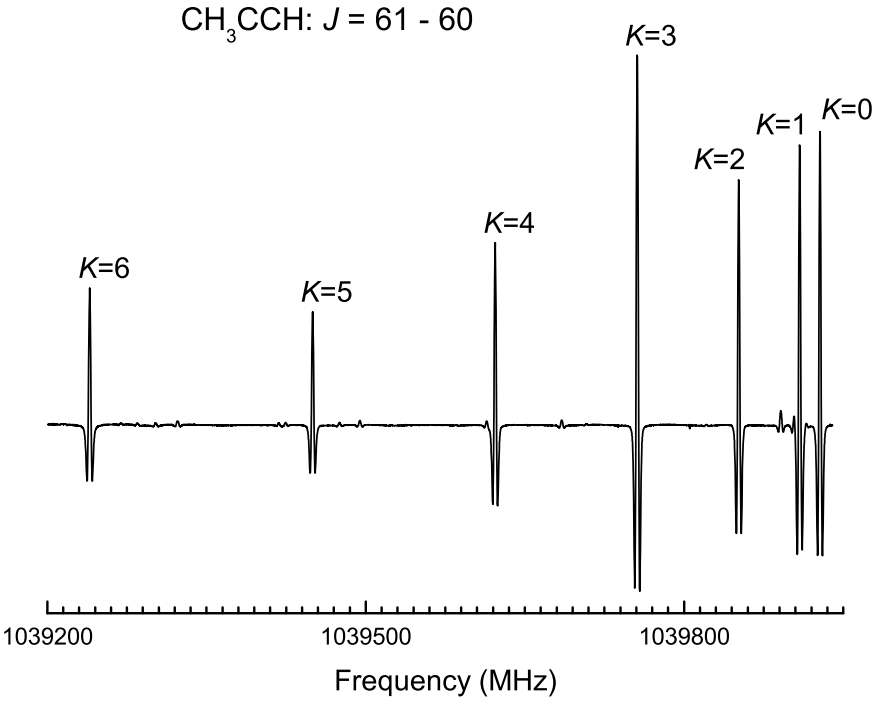

Fig. 1. The $K$-structure, with $K$ ranging from 0 to 6 , of the $J=61 \leftarrow$ 60 transition of $\mathrm{CH}_{3} \mathrm{CCH}$.

set available, the ground-state rotational spectrum of methylacetylene was investigated in the submillimeter-wave region by Müller et al. (2002), whereas that of methyldiacetylene was accurately measured in the $8-224 \mathrm{GHz}$ frequency range by Bester et al. (1984). To our knowledge, the latter are the most recent laboratory investigations carried out for the main isotopic species of 


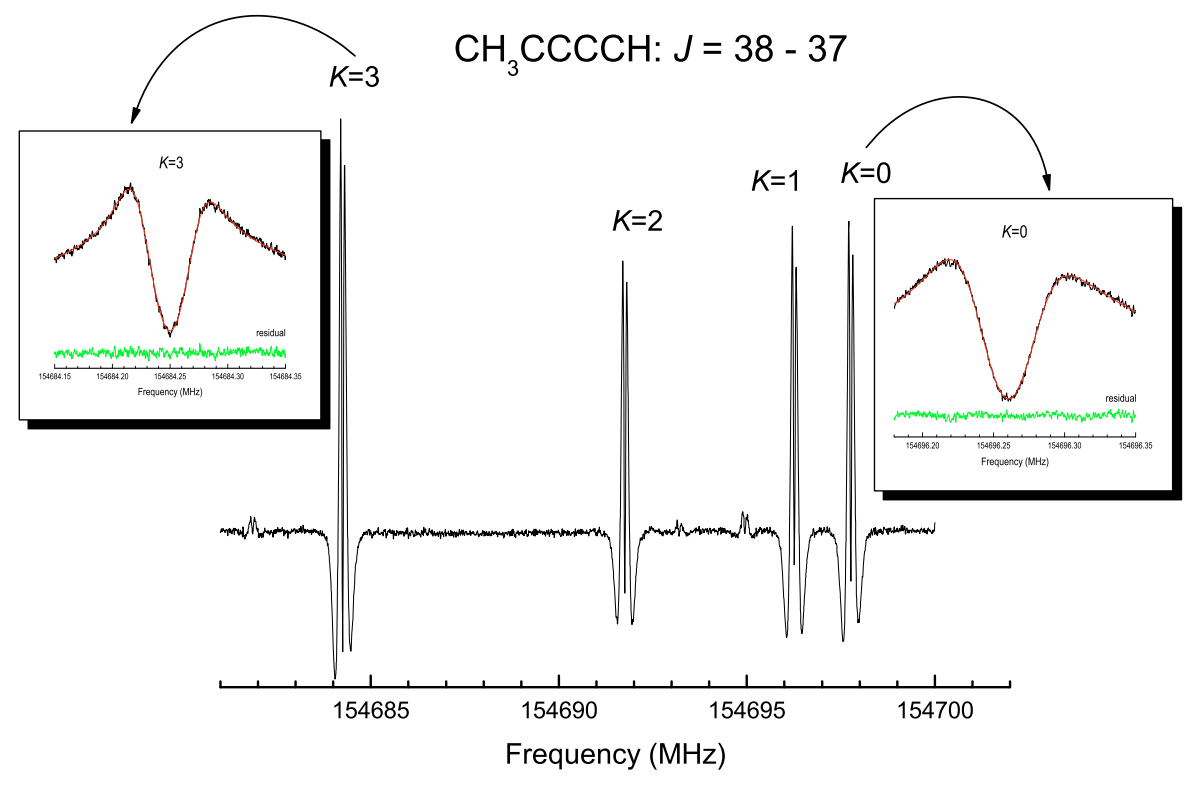

Fig. 2. The $J=38 \leftarrow 37, K=0-3$, transition of $\mathrm{CH}_{3} \mathrm{CCCCH}$ recorded at sub-Doppler resolution. In the insets: the line profile analyzes performed for the $K=0$ and $K=$ 3 components.
$\mathrm{CH}_{3} \mathrm{CCH}$ and $\mathrm{CH}_{3} \mathrm{CCCCH}$, respectively. As far as methylacetylene is concerned, it should be noted that this molecule was extensively studied in the past, and we refer the reader to Müller et al. (2002) for a comprehensive summary. Here, we only recall that Wlodarczak et al. (1988) performed the first investigation of the submillimeter-wave rotational spectrum of methylacetylene, Graner et al. (1989) carried out the first precise determination of the $A_{0}$ rotational constant, and Urban et al. (1995) obtained reliable ground-state rotational energies by means of conventional, as well as Padé approximant approaches.

To improve even more the spectroscopic parameters of methylacetylene and methyldiacetylene and to provide accurate rest frequencies for both of them, for the first time in the present investigation we performed precise Lamb-dip laboratory spectroscopy, which allows transition frequencies to be obtained with an accuracy as good as $0.5-1 \mathrm{kHz}$. In addition, measurements in the $\mathrm{THz}$ frequency region were also carried out for $\mathrm{CH}_{3} \mathrm{CCH}$.

\section{Experimental details and spectra analysis}

In the present investigation, Lamb-dip measurements were performed employing a frequency-modulated, computer-controlled spectrometer in conjunction with a conventional free space cell, as shown in Cazzoli \& Dore (1990a). To increase the sensitivity of the spectrometer as well as of the Lamb-dip effect the radiation path has been doubled (Dore et al. 1999). A detailed description of the spectrometer is given in Cazzoli \& Dore (1990b), whereas our experimental set up for performing sub-Doppler resolution spectroscopy has been described in some previous papers (see for examples, Cazzoli et al. 2002a,b, 2003). Therefore, here, we only report the main details related to the present investigation. The millimeter and submillimeter-wave sources employed are either frequency multipliers driven by gunn diode oscillators or gunn diodes themselves covering, on the whole, the $80-750 \mathrm{GHz}$ frequency range. The frequency modulation is obtained by sine-wave modulating the $73 \mathrm{MHz}$ local oscillator of the synchronization loop at $1.666 \mathrm{kHz}$. A liquid $\mathrm{He}$-cooled $\mathrm{InSb}$ detector was used, and its output was processed by means of a Lock-in amplifier tuned at twice the modulation frequency, so that the second derivative of the natural line profile was recorded.

In addition, Doppler-limited measurements were also carried out for methylacetylene in the 1.0-1.2 THz frequency range.
In particular, the $\mathrm{THz}$ frequency source is actually a tripler followed by another tripler (RPG GmbH), whose radiation power is on the whole 10 to $40 \mu \mathrm{W}$. A liquid He-cooled Ge bolometer was employed as a detector in the $\mathrm{THz}$ region. Another difference concerns the radiation path. In fact, in the $\mathrm{THz}$ region parabolic mirrors, instead of high density polyethylene lenses, were used for collimating and focalizing the radiation. The frequency modulation has been obtained by sine-wave modulating the $73 \mathrm{MHz}$ local oscillator of the synchronization loop at $333 \mathrm{~Hz}$. For both Lamb-dip and $\mathrm{THz}$ measurements, the frequency sources are phase-locked to a Rubidium frequency standard.

The Lamb-dip measurements were carried out at pressures of 0.3-1 mTorr. Such low values of working pressure were chosen to minimize the dip widths as much as possible, as well as to avoid pressure frequency shift effects. In addition, the source power was adequately reduced to have narrow and not distorted dips. The modulation depth used was adjusted in the ranges of 16 to $32 \mathrm{kHz}$ according to the experimental conditions and the transitions under consideration. As far as the Doppler-limited measurements are concerned, higher values of pressure (i.e., 25-90 mTorr) and modulation depth (i.e., 450-1800 kHz) were used. All measurements have been carried out at room temperature. A commercial sample of methylacetylene was used without any further purifications, whereas methyldiacetylene was synthesized by following the procedure reported in Verkruijsse \& Brandsma (1991), as described in Cazzoli et al. (2008).

Going into more detail, the $K$ structures, with $K$ ranging from 0 to 15 , of the $J+1 \leftarrow J$ rotational transitions, with $J=$ $8,9,11,26,29,39,41,60-62,65,67,69$ for methylacetylene and $J=19,24,37,42,50,74,84,95,113,125$ for methyldiacetylene, were recorded with an accuracy of about $1 \mathrm{kHz}$ for the Lamb-dip measurements and $10-20 \mathrm{kHz}$ for the Dopplerlimited ones.

Figure 1 presents an example of $\mathrm{THz}$ measurements: the $K$-components, with $K$ ranging from 0 to 6 , of the $J=61 \leftarrow$ 60 transition of $\mathrm{CH}_{3} \mathrm{CCH}$. It is evident that the $K=3 n$ lines are more intense than the $K \neq 3 n$ ones due to the hydrogen nuclear statistics. It is also worthwhile noting the extremely good signal-to-noise ratio of the recorded spectrum that actually allowed a frequency accuracy as good as $20 \mathrm{kHz}$. In Fig. 2 the $J=38 \leftarrow 37, K=0-3$, transition of $\mathrm{CH}_{3} \mathrm{CCCCH}$ is depicted providing an example of rotational spectrum recorded at 
Table 1. Rotational $J^{\prime}, K^{\prime} \leftarrow J^{\prime \prime}, K^{\prime \prime}$ transitions $(\mathrm{MHz})$ of $\mathrm{CH}_{3} \mathrm{CCH}$ with the corresponding observed-calculated (o-c) differences (kHz) (second fit: see text).

\begin{tabular}{|c|c|c|c|c|c|c|c|c|c|c|c|}
\hline \multicolumn{4}{|c|}{ Transition } & \multirow{2}{*}{$\begin{array}{l}\text { Freq. } \\
\text { (MHz) }\end{array}$} & \multirow{2}{*}{$\begin{array}{c}\mathrm{O}-\mathrm{c} \\
(\mathrm{kHz})\end{array}$} & \multicolumn{4}{|c|}{ Transition } & \multirow{2}{*}{$\begin{array}{l}\text { Freq. } \\
\text { (MHz) }\end{array}$} & \multirow{2}{*}{$\begin{array}{c}\mathrm{O}-\mathrm{c} \\
(\mathrm{kHz})\end{array}$} \\
\hline$J^{\prime}$ & $K^{\prime}$ & $J^{\prime \prime}$ & $K^{\prime \prime}$ & & & $J^{\prime}$ & $K^{\prime}$ & $J^{\prime \prime}$ & $K^{\prime \prime}$ & & \\
\hline 9 & 8 & 8 & 8 & $153629.5158(5)$ & 0.5 & 42 & 9 & 41 & 9 & $715895.0461(5)$ & 0.1 \\
\hline 9 & 7 & 8 & 7 & $153673.4387(5)$ & 0.0 & 42 & 8 & 41 & 8 & $716122.8242(5)$ & 0.9 \\
\hline 9 & 6 & 8 & 6 & $153711.5389(5)$ & 0.4 & 42 & 6 & 41 & 6 & $716498.5235(5)$ & 0.3 \\
\hline 9 & 5 & 8 & 5 & $153743.8027(5)$ & -0.1 & 42 & 5 & 41 & 5 & $716646.3035(5)$ & 0.3 \\
\hline 9 & 4 & 8 & 4 & $153770.2187(5)$ & 0.0 & 42 & 4 & 41 & 4 & 716 767.2919(5) & 0.4 \\
\hline 9 & 3 & 8 & 3 & $153790.7720(5)$ & 0.9 & 42 & 3 & 41 & 3 & $716861.4423(5)$ & 0.6 \\
\hline 9 & 2 & 8 & 2 & $153805.4627(5)$ & 1.0 & 42 & 2 & 41 & 2 & $716928.7179(5)$ & 0.1 \\
\hline 9 & 1 & 8 & 1 & $153814.2764(5)$ & 0.8 & 42 & 1 & 41 & 1 & $716969.0939(5)$ & 0.2 \\
\hline 9 & 0 & 8 & 0 & $153817.2147(5)$ & 0.3 & 42 & 0 & 41 & 0 & $716982.5547(5)$ & 0.6 \\
\hline 10 & 9 & 9 & 9 & $170642.0339(5)$ & 0.0 & 61 & 12 & 60 & 12 & $1037184.554(20)$ & 10.4 \\
\hline 10 & 8 & 9 & 8 & $170697.2742(5)$ & 0.1 & 61 & 9 & 60 & 9 & $1038381.782(20)$ & 9.7 \\
\hline 10 & 7 & 9 & 7 & $170746.0668(5)$ & 0.2 & 61 & 8 & 60 & 8 & $1038705.667(20)$ & 3.6 \\
\hline 10 & 6 & 9 & 6 & $170788.3907(5)$ & -0.9 & 61 & 7 & 60 & 7 & $1038991.745(20)$ & 4.8 \\
\hline 10 & 5 & 9 & 5 & $170824.2315(5)$ & -1.0 & 61 & 3 & 60 & 3 & $1039755.868(20)$ & -13.8 \\
\hline 10 & 4 & 9 & 4 & $170853.5757(5)$ & 0.2 & 61 & 2 & 60 & 2 & $1039851.520(20)$ & -17.5 \\
\hline 10 & 3 & 9 & 3 & $170876.4101(5)$ & 0.8 & 61 & 1 & 60 & 1 & $1039908.920(20)$ & -2.1 \\
\hline 10 & 2 & 9 & 2 & $170892.7264(5)$ & 0.4 & 61 & 0 & 60 & 0 & $1039928.059(20)$ & 21.5 \\
\hline 10 & 1 & 9 & 1 & $170902.5184(5)$ & 1.0 & 62 & 15 & 61 & 15 & $1052546.059(20)$ & 10.9 \\
\hline 10 & 0 & 9 & 0 & $170905.7826(5)$ & 0.2 & 62 & 14 & 61 & 14 & $1053102.081(20)$ & -1.4 \\
\hline 12 & 11 & 11 & 11 & $204608.7740(5)$ & -0.3 & 62 & 13 & 61 & 13 & $1053620.666(20)$ & 7.7 \\
\hline 12 & 10 & 11 & 10 & $204690.4219(5)$ & 0.7 & 62 & 12 & 61 & 12 & 1054 101.576(20) & -14.0 \\
\hline 12 & 9 & 11 & 9 & $204764.3869(5)$ & 0.9 & 62 & 9 & 61 & 9 & $1055316.849(20)$ & 1.7 \\
\hline 12 & 8 & 11 & 8 & $204830.6433(5)$ & -1.6 & 62 & 6 & 61 & 6 & $1056187.810(20)$ & -9.6 \\
\hline 12 & 7 & 11 & 7 & $204889.1662(5)$ & -0.9 & 62 & 5 & 61 & 5 & $1056401.104(20)$ & 4.9 \\
\hline 12 & 6 & 11 & 6 & $204939.9313(5)$ & -0.8 & 62 & 4 & 61 & 4 & $1056575.711(20)$ & 0.5 \\
\hline 12 & 5 & 11 & 5 & $204982.9186(5)$ & -0.6 & 62 & 3 & 61 & 3 & $1056711.590(20)$ & 1.9 \\
\hline 12 & 4 & 11 & 4 & $205018.1137(5)$ & 0.9 & 62 & 2 & 61 & 2 & $1056808.679(20)$ & -1.3 \\
\hline 12 & 3 & 11 & 3 & $205045.5006(5)$ & -1.0 & 62 & 1 & 61 & 1 & $1056866.942(20)$ & -8.4 \\
\hline 12 & 2 & 11 & 2 & $205065.0702(5)$ & 1.6 & 62 & 0 & 61 & 0 & $1056886.392(20)$ & 15.7 \\
\hline 12 & 1 & 11 & 1 & $205076.8164(5)$ & 0.2 & 63 & 15 & 62 & 15 & $1069435.901(20)$ & 22.4 \\
\hline 12 & 0 & 11 & 0 & $205080.7322(5)$ & -0.1 & 63 & 14 & 62 & 14 & $1070000.134(20)$ & 3.9 \\
\hline 27 & 12 & 26 & 12 & $459991.3044(5)$ & 0.8 & 63 & 12 & 62 & 12 & $1071014.381(20)$ & -24.8 \\
\hline 27 & 10 & 26 & 10 & $460373.4414(5)$ & -0.1 & 63 & 9 & 62 & 9 & $1072247.600(20)$ & -15.1 \\
\hline 27 & 9 & 26 & 9 & $460538.7862(5)$ & 0.2 & 63 & 6 & 62 & 6 & $1073131.464(20)$ & 12.9 \\
\hline 27 & 8 & 26 & 8 & $460686.8956(5)$ & 0.0 & 63 & 3 & 62 & 3 & $1073662.955(20)$ & 0.9 \\
\hline 27 & 7 & 26 & 7 & $460817.7142(5)$ & -0.2 & 63 & 2 & 62 & 2 & $1073761.500(20)$ & 20.0 \\
\hline 27 & 6 & 26 & 6 & $460931.1924(5)$ & 0.0 & 63 & 1 & 62 & 1 & $1073820.627(20)$ & 16.5 \\
\hline 27 & 5 & 26 & 5 & $461027.2859(5)$ & -0.4 & 63 & 0 & 62 & 0 & $1073840.335(20)$ & 11.8 \\
\hline 27 & 4 & 26 & 4 & 461 105.9587(5) & -0.5 & 66 & 15 & 65 & 15 & $1120080.104(20)$ & 85.2 \\
\hline 27 & 3 & 26 & 3 & 461 167.1808(5) & 0.0 & 66 & 14 & 65 & 14 & $1120668.720(20)$ & 3.2 \\
\hline 27 & 2 & 26 & 2 & $461210.9275(5)$ & 0.1 & 66 & 12 & 65 & 12 & $1121726.916(20)$ & -12.6 \\
\hline 27 & 1 & 26 & 1 & $461237.1825(5)$ & 0.3 & 66 & 9 & 65 & 9 & $1123013.530(20)$ & -14.3 \\
\hline 27 & 0 & 26 & 0 & $461245.9347(5)$ & -0.1 & 66 & 6 & 65 & 6 & $1123935.641(20)$ & -6.6 \\
\hline 30 & 12 & 29 & 12 & $511043.7708(5)$ & 0.2 & 66 & 3 & 65 & 3 & $1124490.150(20)$ & -9.6 \\
\hline 30 & 9 & 29 & 9 & $511650.9347(5)$ & 0.5 & 66 & 2 & 65 & 2 & $1124592.962(20)$ & 11.4 \\
\hline 30 & 6 & 29 & 6 & $512086.1146(5)$ & -0.7 & 66 & 1 & 65 & 1 & $1124654.668(20)$ & 27.3 \\
\hline 30 & 5 & 29 & 5 & $512192.6838(5)$ & -0.1 & 66 & 0 & 65 & 0 & $1124675.193(20)$ & -13.6 \\
\hline 30 & 4 & 29 & 4 & $512279.9320(5)$ & -0.4 & 68 & 15 & 67 & 15 & $1153821.001(20)$ & -32.8 \\
\hline 30 & 3 & 29 & 3 & $512347.8264(5)$ & -0.8 & 68 & 14 & 67 & 14 & $1154425.770(20)$ & 14.2 \\
\hline 30 & 2 & 29 & 2 & $512396.3417(5)$ & -0.6 & 68 & 12 & 67 & 12 & $1155512.975(20)$ & -12.3 \\
\hline 30 & 1 & 29 & 1 & $512425.4585(5)$ & -0.4 & 68 & 9 & 67 & 9 & $1156834.790(20)$ & -23.6 \\
\hline 30 & 0 & 29 & 0 & $512435.1651(5)$ & -0.6 & 68 & 6 & 67 & 6 & $1157782.140(20)$ & -16.4 \\
\hline 40 & 15 & 39 & 15 & $680048.5349(5)$ & -0.5 & 68 & 3 & 67 & 3 & $1158351.831(20)$ & -21.3 \\
\hline 40 & 12 & 39 & 12 & $681076.7384(5)$ & -0.1 & 68 & 2 & 67 & 2 & $1158457.443(20)$ & -19.2 \\
\hline 40 & 9 & 39 & 9 & $681880.0715(5)$ & -0.2 & 68 & 1 & 67 & 1 & $1158520.829(20)$ & -26.5 \\
\hline 40 & 6 & 39 & 6 & $682455.8480(5)$ & -0.1 & 68 & 0 & 67 & 0 & $1158541.958(20)$ & 25.8 \\
\hline 40 & 5 & 39 & 5 & $682596.8450(5)$ & 0.0 & 70 & 15 & 69 & 15 & $1187543.822(20)$ & -26.4 \\
\hline 40 & 4 & 39 & 4 & $682712.2799(5)$ & 0.0 & 70 & 12 & 69 & 12 & $1189280.580(20)$ & -10.2 \\
\hline 40 & 3 & 39 & 3 & $682802.1084(5)$ & -0.3 & 70 & 9 & 69 & 9 & $1190637.299(20)$ & -32.5 \\
\hline 40 & 2 & 39 & 2 & $682866.2964(5)$ & -0.5 & 70 & 6 & 69 & 6 & $1191609.639(20)$ & -22.5 \\
\hline 40 & 1 & 39 & 1 & $682904.8193(5)$ & -0.3 & 70 & 3 & 69 & 3 & $1192194.370(20)$ & -17.2 \\
\hline 40 & 0 & 39 & 0 & $682917.6621(5)$ & -0.1 & 70 & 2 & 69 & 2 & $1192302.756(20)$ & -8.7 \\
\hline 42 & 15 & 41 & 15 & $713975.3826(5)$ & 0.4 & 70 & 1 & 69 & 1 & $1192367.809(20)$ & -0.7 \\
\hline 42 & 12 & 41 & 12 & $715053.0606(5)$ & -0.6 & 70 & 0 & 69 & 0 & $1192389.488(20)$ & -0.4 \\
\hline
\end{tabular}


Table 2. Rotational $J^{\prime}, K^{\prime} \leftarrow J^{\prime \prime}, K^{\prime \prime}$ transitions (MHz) of $\mathrm{CH}_{3} \mathrm{CCCCH}$ with the corresponding observed-calculated (o-c) differences (kHz).

\begin{tabular}{|c|c|c|c|c|c|c|c|c|c|c|c|}
\hline \multicolumn{4}{|c|}{ Transition } & \multirow{2}{*}{$\begin{array}{l}\text { Freq. } \\
(\mathrm{MHz})\end{array}$} & \multirow{2}{*}{$\begin{array}{c}\mathrm{O}-\mathrm{c} \\
(\mathrm{kHz})\end{array}$} & \multicolumn{4}{|c|}{ Transition } & \multirow{2}{*}{$\begin{array}{l}\text { Freq. } \\
(\mathrm{MHz})\end{array}$} & \multirow{2}{*}{$\begin{array}{c}\mathrm{o}-\mathrm{c} \\
(\mathrm{kHz})\end{array}$} \\
\hline$J^{\prime}$ & $K^{\prime}$ & $J^{\prime \prime}$ & $K^{\prime \prime}$ & & & $J^{\prime}$ & $K^{\prime}$ & $J^{\prime \prime}$ & $K^{\prime \prime}$ & & \\
\hline 20 & $\overline{10}$ & 19 & $\overline{10}$ & $81347.8940(20)$ & -2.2 & 51 & 5 & 50 & 5 & $207550.0766(10)$ & -0.6 \\
\hline 20 & 9 & 19 & 9 & $81362.9239(20)$ & -1.8 & 51 & 4 & 50 & 4 & $207568.1301(10)$ & 1.2 \\
\hline 20 & 8 & 19 & 8 & $81376.3845(20)$ & 2.8 & 51 & 3 & 50 & 3 & $207582.1736(10)$ & 0.7 \\
\hline 20 & 6 & 19 & 6 & $81398.5597(10)$ & -1.7 & 51 & 2 & 50 & 2 & $207592.2045(10)$ & -1.8 \\
\hline 20 & 5 & 19 & 5 & $81407.2797(10)$ & -1.1 & 51 & 1 & 50 & 1 & $207598.2263(10)$ & -0.9 \\
\hline 20 & 4 & 19 & 4 & $81414.4154(10)$ & -1.9 & 51 & 0 & 50 & 0 & $207600.2340(10)$ & -0.3 \\
\hline 20 & 3 & 19 & 3 & $81419.9683(10)$ & -1.1 & 75 & 10 & 74 & 10 & $304924.4685(10)$ & 1.4 \\
\hline 20 & 2 & 19 & 2 & $81423.9357(10)$ & -0.4 & 75 & 9 & 74 & 9 & $304979.7649(10)$ & 0.9 \\
\hline 20 & 1 & 19 & 1 & $81426.3162(10)$ & -0.2 & 75 & 8 & 74 & 8 & $305029.2716(10)$ & 1.0 \\
\hline 20 & 0 & 19 & 0 & $81427.1083(10)$ & -1.6 & 75 & 7 & 74 & 7 & $305072.9775(10)$ & 0.8 \\
\hline 25 & 9 & 24 & 9 & $101701.7729(10)$ & -1.3 & 75 & 6 & 74 & 6 & $305110.8738(10)$ & 0.4 \\
\hline 25 & 8 & 24 & 8 & $101718.5795(10)$ & -0.6 & 75 & 5 & 74 & 5 & $305142.9538(10)$ & 0.8 \\
\hline 25 & 7 & 24 & 7 & $101733.4147(10)$ & -2.3 & 75 & 4 & 74 & 4 & $305169.2097(10)$ & 0.8 \\
\hline 25 & 6 & 24 & 6 & $101746.2812(10)$ & -0.7 & 75 & 3 & 74 & 3 & $305189.6366(10)$ & 0.9 \\
\hline 25 & 5 & 24 & 5 & $101757.1710(10)$ & -1.1 & 75 & 2 & 74 & 2 & $305204.2301(10)$ & 0.8 \\
\hline 25 & 4 & 24 & 4 & $101766.0833(10)$ & -2.1 & 75 & 1 & 74 & 1 & $305212.9874(10)$ & 0.8 \\
\hline 25 & 3 & 24 & 3 & $101773.0196(10)$ & -0.2 & 75 & 0 & 74 & 0 & $305215.9069(10)$ & 1.0 \\
\hline 25 & 2 & 24 & 2 & $101777.9727(10)$ & -1.3 & 85 & 12 & 84 & 12 & $345391.7325(10)$ & 0.8 \\
\hline 25 & 1 & 24 & 1 & $101780.9450(10)$ & -1.9 & 85 & 11 & 84 & 11 & $345467.0523(10)$ & 0.7 \\
\hline 25 & 0 & 24 & 0 & $101781.9362(10)$ & -1.8 & 85 & 10 & 84 & 10 & $345535.8733(10)$ & -0.1 \\
\hline 38 & 12 & 37 & 12 & $154482.0271(10)$ & 0.3 & 85 & 9 & 84 & 9 & $345598.1832(10)$ & 0.0 \\
\hline 38 & 11 & 37 & 11 & $154516.4115(10)$ & -0.7 & 85 & 8 & 84 & 8 & $345653.9676(10)$ & -0.4 \\
\hline 38 & 9 & 37 & 9 & $154576.2795(10)$ & 1.0 & 85 & 7 & 84 & 7 & $345703.2163(10)$ & -0.5 \\
\hline 38 & 8 & 37 & 8 & $154601.7462(10)$ & -0.4 & 85 & 6 & 84 & 6 & $345745.9192(10)$ & -0.1 \\
\hline 38 & 7 & 37 & 7 & $154624.2323(10)$ & 1.3 & 85 & 5 & 84 & 5 & $345782.0663(10)$ & -0.6 \\
\hline 38 & 6 & 37 & 6 & $154643.7276(10)$ & 0.8 & 85 & 4 & 84 & 4 & $345811.6518(10)$ & -0.4 \\
\hline 38 & 5 & 37 & 5 & $154660.2304(10)$ & 0.2 & 85 & 3 & 84 & 3 & $345834.6691(10)$ & -0.1 \\
\hline 38 & 4 & 37 & 4 & $154673.7363(10)$ & -1.1 & 85 & 2 & 84 & 2 & $345851.1129(10)$ & -0.4 \\
\hline 38 & 3 & 37 & 3 & $154684.2471(10)$ & 0.9 & 85 & 1 & 84 & 1 & $345860.9817(10)$ & 0.5 \\
\hline 38 & 2 & 37 & 2 & $154691.7525(10)$ & -1.3 & 85 & 0 & 84 & 0 & $345864.2708(10)$ & 0.1 \\
\hline 38 & 1 & 37 & 1 & 154 696.2587(10) & -0.3 & 96 & 12 & 95 & 12 & $390027.1125(20)$ & -0.6 \\
\hline 38 & 0 & 37 & 0 & $154697.7624(10)$ & 1.4 & 96 & 11 & 95 & 11 & $390111.5765(20)$ & -0.9 \\
\hline 43 & 12 & 42 & 12 & $174802.9361(10)$ & -0.9 & 96 & 10 & 95 & 10 & $390188.7530(10)$ & -1.3 \\
\hline 43 & 11 & 42 & 11 & 174 841.7894(10) & 0.0 & 96 & 9 & 95 & 9 & $390258.6272(10)$ & -1.1 \\
\hline 43 & 10 & 42 & 10 & $174877.2910(10)$ & 0.7 & 96 & 8 & 95 & 8 & $390321.1850(10)$ & -0.1 \\
\hline 43 & 9 & 42 & 9 & $174909.4335(10)$ & 1.2 & 96 & 7 & 95 & 7 & $390376.4117(10)$ & -0.3 \\
\hline 43 & 8 & 42 & 8 & $174938.2106(10)$ & 1.8 & 96 & 5 & 95 & 5 & $390464.8311(10)$ & -2.2 \\
\hline 43 & 7 & 42 & 7 & $174963.6153(10)$ & 1.5 & 96 & 4 & 95 & 4 & $390498.0103(10)$ & 0.5 \\
\hline 43 & 6 & 42 & 6 & $174985.6430(10)$ & 0.8 & 96 & 3 & 95 & 3 & $390523.8210(10)$ & 0.4 \\
\hline 43 & 5 & 42 & 5 & $175004.2895(10)$ & 0.3 & 96 & 2 & 95 & 2 & $390542.2616(10)$ & 0.9 \\
\hline 43 & 4 & 42 & 4 & 175 019.5537(10) & 2.5 & 96 & 1 & 95 & 1 & $390553.3245(10)$ & -1.6 \\
\hline 43 & 3 & 42 & 3 & $175031.4259(10)$ & 1.0 & 96 & 0 & 95 & 0 & $390557.0147(10)$ & -0.1 \\
\hline 43 & 2 & 42 & 2 & $175039.9090(10)$ & 1.2 & 114 & 3 & 113 & 3 & $463598.4658(10)$ & -0.3 \\
\hline 43 & 1 & 42 & 1 & $175044.9993(10)$ & 1.0 & 114 & 2 & 113 & 2 & $463620.0707(10)$ & -1.5 \\
\hline 43 & 0 & 42 & 0 & $175046.6962(10)$ & 1.0 & 114 & 1 & 113 & 1 & $463633.0372(10)$ & -0.3 \\
\hline 51 & 12 & 50 & 12 & $207311.9202(20)$ & 0.0 & 114 & 0 & 113 & 0 & $463637.3598(10)$ & 0.1 \\
\hline 51 & 11 & 50 & 11 & $207357.8740(20)$ & -0.5 & 126 & 6 & 125 & 6 & $512145.6527(10)$ & 1.3 \\
\hline 51 & 10 & 50 & 10 & $207399.8637(20)$ & -0.9 & 126 & 3 & 125 & 3 & $512273.2460(10)$ & -0.1 \\
\hline 51 & 9 & 50 & 9 & $207437.8801(10)$ & -1.7 & 126 & 2 & 125 & 2 & $512296.8883(10)$ & 1.0 \\
\hline 51 & 8 & 50 & 8 & $207471.9164(10)$ & -1.9 & 126 & 1 & 125 & 1 & $512311.0737(10)$ & -0.1 \\
\hline 51 & 7 & 50 & 7 & $207501.9661(10)$ & -0.9 & 126 & 0 & 125 & 0 & $512315.8030(10)$ & 0.0 \\
\hline 51 & 6 & 50 & 6 & $207528.0226(10)$ & 0.7 & & & & & & \\
\hline
\end{tabular}

sub-Doppler resolution. This figure, even if recorded with a modulation depth greater than that used for resolving the individual $K$ components (shown as examples in the insets for $K=$ 0 and 3), allows us to point out that transition frequencies can be more accurately retrieved by employing the Lamb-dip technique thanks to the narrower line width.

\section{Analysis and results}

In analyzing the spectra, we note that in no cases has the hyperfine structure due to the hydrogen nuclei been resolved. The transition frequencies were determined either by fitting the experimental data points to a parabolic function or by a line profile analysis (see Cazzoli \& Dore 1990a), with the former mostly employed and the latter mainly used for distorted dips. A graphical example of the line profile analysis performed is given in the insets of Fig. 2. In all cases the reported frequencies are obtained as averages of a set of measurements, which were then very reproducible, i.e., the frequency differences are less (or even much less) than $1 \mathrm{kHz}$. Therefore, the standard deviation of the mean was used as indicator of the frequency accuracy. Consequently, the derived rest frequencies have an estimated accuracy of 0.5 
Table 3. Ground-state rotational and centrifugal distortion constants of $\mathrm{CH}_{3} \mathrm{CCH}$ and $\mathrm{CH}_{3} \mathrm{CCCCH}$.

\begin{tabular}{|c|c|c|c|c|c|c|}
\hline \multicolumn{7}{|l|}{ 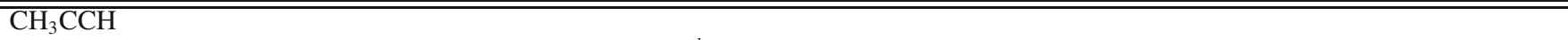 } \\
\hline & & This work $^{a}$ & This work $^{b}$ & This work $^{c}$ & Müller et al. (2002) & Müller et al. (2000) \\
\hline$A_{0}-B_{0}$ & $(\mathrm{MHz})$ & $\overline{-}$ & - & $150594.45(55)$ & $150594.45(46)$ & \\
\hline$B_{0}$ & $(\mathrm{MHz})$ & $8545.8770201(87)$ & $8545.8769958(63)$ & $8545.8769857(60)$ & $8545.8768621(217)$ & $8545.8768831(220)$ \\
\hline$D_{J}$ & $(\mathrm{kHz})$ & $2.9393482(86)$ & $2.9393197(40)$ & $2.9393130(32)$ & $2.9392410(251)$ & $2.9393443(316)$ \\
\hline$D_{J K}$ & $(\mathrm{kHz})$ & $163.41736(25)$ & $163.41699(22)$ & $163.41667(21)$ & 163.41449(209) & $163.41306(227)$ \\
\hline$D_{K}$ & $(\mathrm{kHz})$ & - & - & $2907.2(140)$ & 2907.2(122) & \\
\hline$H_{J}$ & $(\mathrm{~Hz})$ & $-0.0000429(25)$ & $-0.0000514(10)$ & $-0.00005251(63)$ & $-0.00006320(610)$ & $-0.00002960(950)$ \\
\hline$H_{J K}$ & $(\mathrm{~Hz})$ & $0.91595(16)$ & $0.91573(15)$ & $0.91551(13)$ & $0.91408(89)$ & $0.91451(113)$ \\
\hline$H_{K J}$ & $(\mathrm{~Hz})$ & $5.2984(22)$ & $5.2974(20)$ & $5.2967(19)$ & $5.3021(114)$ & $5.2914(116)$ \\
\hline$L_{J J K}$ & $(\mathrm{mHz})$ & $-0.006629(52)$ & $-0.006672(61)$ & $-0.006641(49)$ & $-0.006313(148)$ & $-0.006588(232)$ \\
\hline$L_{J K}$ & $(\mathrm{mHz})$ & $-0.04405(72)$ & $-0.04341(56)$ & $-0.04228(42)$ & $-0.04181(189)$ & $-0.03915(223)$ \\
\hline$L_{K K J}$ & $(\mathrm{mHz})$ & $-0.3770(66)$ & $-0.3802(61)$ & $-0.3899(57)$ & $-0.4194(203)$ & $-0.4171(214)$ \\
\hline$P_{J J J J K}$ & $(\mu \mathrm{Hz})$ & - & $0.0000340(64)$ & $0.0000297(52)$ & & \\
\hline rms error $^{d}$ & $(\mathrm{MHz})$ & 0.00056 & 0.0126 & 23.5 & & \\
\hline \multicolumn{7}{|c|}{$\mathrm{CH}_{3} \mathrm{CCCCH}$} \\
\hline & & This work $^{a}$ & Bester et al. (1984) & & & \\
\hline$B_{0}$ & $(\mathrm{MHz})$ & $2035.7470641(46)$ & $2035.74706(8)$ & & & \\
\hline$D_{J}$ & $(\mathrm{kHz})$ & $0.08664738(54)$ & $0.086687(40)$ & & & \\
\hline$D_{J K}$ & $(\mathrm{kHz})$ & $19.86699(20)$ & $19.8630(12)$ & & & \\
\hline$H_{J}$ & $(\mathrm{~Hz})$ & $0.000004472(18)$ & $0.0000130(66)$ & & & \\
\hline$H_{J K}$ & $(\mathrm{~Hz})$ & $0.036688(21)$ & $0.03568(21)$ & & & \\
\hline$H_{K J}$ & $(\mathrm{~Hz})$ & $0.3435(13)$ & $0.3278(37)$ & & & \\
\hline$L_{J J K}$ & $(\mathrm{mHz})$ & $-0.00008561(68)$ & & & & \\
\hline$L_{J K}$ & $(\mathrm{mHz})$ & $-0.00109(10)$ & & & & \\
\hline rms error $^{d}$ & $(\mathrm{kHz})$ & 1.1 & & & & \\
\hline
\end{tabular}

${ }^{a}$ Fit performed including only Lamb-dip measurements, ${ }^{b}$ fit performed including Lamb-dip and THz measurements. The corresponding residuals have been reported in Table 1, ${ }^{c}$ fit performed including Lamb-dip, THz, $\Delta K=3$ (Müller et al. 2002) and other measurements available (see text), ${ }^{d}$ root mean square error of the fit as provided by the SPFIT/SPCAT program.

and $1 \mathrm{kHz}$ for methylacetylene and methyldiacetylene, respectively. An uncertainty of $2 \mathrm{kHz}$ has only been assigned to the weaker transitions of $\mathrm{CH}_{3} \mathrm{CCCCH}$. In the case of methylacetylene, the high reproducibility of the Lamb-dip measurements is mainly due to the fact that the dips are particularly narrow, narrower than for methyldiacetylene. This may be ascribed to the different dipole moments: 0.7804(1) D for $\mathrm{CH}_{3} \mathrm{CCH}$ (Muenter \& Laurie 1966) and 1.2071(10) D (Bester et al. 1984), consequently to a lower relaxation rate for $\mathrm{CH}_{3} \mathrm{CCH}$. Finally, for Doppler-limited measurements, the frequencies were only obtained by fitting the experimental data points to a parabolic function.

On the whole, a total of 128 and 103 distinct frequency lines have been assigned for $\mathrm{CH}_{3} \mathrm{CCH}$ and $\mathrm{CH}_{3} \mathrm{CCCCH}$, respectively. They are collected in Tables 1 (methylacetylene) and 2 (methyldiacetylene), together with the corresponding residuals (obs. calc.) of the fit described below. We may notice that they are lower in almost all cases than the given uncertainties.

For both molecules, the retrieved frequency values were included in least-squares fits in which each line frequency was weighted proportionally to the inverse square of its experimental uncertainty. For $\mathrm{CH}_{3} \mathrm{CCH}$, three different fits were carried out: the first one including only Lamb-dip measurements, the second including both Lamb-dip and $\mathrm{THz}$ measurements, and a third one in which, in addition to our transition frequencies, data available in the literature have also been included in the analysis. More precisely, some accurate low-frequency values (i.e., <150 GHz) (Dubrulle et al. 1978; Ware \& Roberts 1984), some transitions with $K$ values greater than 15 (Müller et al. 2002), some transitions in the $720-1000 \mathrm{GHz}$ frequency range (Müller et al. 2002) and, finally, $\Delta K=+3$ ground-state combination differences (Müller et al. 2002) have been considered. The last allows determination of the axial $A_{0}$ and $D_{K}$ constants.
All these fits were carried out with Pickett's SPCAT/SPFIT suite of programs (Pickett 1991). The results are reported in Table 3 for both methylacetylene and methyldiacetylene. In both cases the present spectroscopic parameters are compared with those available in the literature. From this comparison, one may first note that our results for both species are in overall good agreement with the most recently published studies, but nevertheless the present work reports clear improvements with respect to the literature data.

As far as methylacetylene is concerned, for the first fit, i.e., the one including only Lamb-dip measurements, the small standard deviation has to be noted; in fact, such a small $\sigma(0.56 \mathrm{kHz})$ points out that the spectroscopic parameters obtained are able to reproduce the rotational frequencies with an accuracy of about $0.5 \mathrm{kHz}$ up to $\sim 700 \mathrm{GHz}$. Therefore, $\mathrm{CH}_{3} \mathrm{CCH}$ might be taken into consideration as secondary frequency standards for the millimeter- and submillimeter-wave (up to $700 \mathrm{GHz}$ ) frequency regions. Concerning the second and third fits, the accuracy of almost all the spectroscopic constants (all but $A_{0}$ and $D_{K}$, in the third fit) has been largely improved by reducing the uncertainties by about one order of magnitude. In addition, our $\mathrm{THz}$ measurements allowed us to determine a new centrifugal distortion constant, the $P_{J J J J K}$. Even larger improvements were obtained for methyldiacetylene, not only were the spectroscopic parameters improved by one or two orders of magnitude, but also two octic centrifugal distortion constants were determined for the first time.

\section{Conclusion}

Precise Lamb-dip measurements were carried out in the 80-750 GHz frequency range for both methylacetylene and methyldiacetylene. They allowed us to provide very accurate 
rest frequencies, as well as to improve the spectroscopic parameters available in the literature. In addition, transition frequencies in the $\mathrm{THz}$ frequency region $(1.0-1.2 \mathrm{THz})$ were recorded at Doppler resolution for $\mathrm{CH}_{3} \mathrm{CCCCH}$.

On the whole, we present the most accurate ground-state rotational parameters of methylacetylene and methyldiacetylene known at the moment. Since both of them are known to have a strong astrophysical relevance, we think that the improvement in the accuracy of the ground-state rotational parameters and of the rest frequencies obtained in the present investigation could be very useful for future observational purposes.

Acknowledgements. This work has been supported by "PRIN 2005" funds (project "Trasferimenti di energia e di carica a livello molecolare") and by University of Bologna (RFO funds).

\section{References}

Agundez, M., Fonfria, J. P., Cernicharo, J., Pardo, J. R., \& Guelin, M. 2008, A\&A, 479, 493

Bester, M., Yamada, K., Winnewisser, G., et al. 1984, A\&A, 137, L20

Cazzoli, G., \& Dore, L. 1990a, J. Mol. Spectrosc., 143, 231

Cazzoli, G., \& Dore, L. 1990b, J. Mol. Spectrosc., 141, 49

Cazzoli, G., Dore, L., Cludi, L., Puzzarini, C., \& Beninati, S. 2002a, J. Mol. Spectrosc., 215, 160

Cazzoli, G., Dore, L., Puzzarini, C., \& Beninati, S. 2002b, Phys. Chem. Chem. Phys., 4, 3575

Cazzoli, G., Puzzarini, C., \& Lapinov, A. V. 2003, ApJ, 592, L95

Cazzoli, G., Cludi, L., Contento, M., \& Puzzarini, C. 2008, J. Mol. Spectrosc., in press
Cernicharo, J., Heras, A. M., Pardo, J. R., et al. 2001, ApJ, 546, L127

Dore, L., Degli Esposti, C., Mazzavillani, A., \& Cazzoli, G. 1999, Chem. Phys. Lett., 300, 489

Dubrulle, A., Boucher, D., Burie, J., \& Demaison, J. 1978, J. Mol. Spectrosc., 72,158

Graner, G., Horneman, V. M., Blanquet, G., et al. 1989, J. Mol. Spectrosc., 135, 32

Loren, R. B., Wootten, A., \& Mundy, L. G. 1984, ApJ, 286, L23

MacLeod, J. M., Avery, L. W., \& Broten, N. W. 1984, ApJ, 282, L89

Mauersberger, R., Henkel, C., Walmsley, C. M., Sage, L. J., \& Wiklind, T. 1991, A\&A, 247, 307

Muenter, J. S., \& Laurie, V. W. 1966, J. Chem. Phys., 45, 855

Müller, H. S. P., Thorwirth, S., Bizzocchi, L., \& Winnewisser, G. 2000, Z. Naturforsch., 55a, 491

Müller, H. S. P., Pracna, P., \& Horneman, V.-M. 2002, J. Mol. Spectrosc., 216, 397

Pickett, H. M. 1991, J. Mol. Spectrosc., 148, 371

Pratap, P., Dickens, J. E., Snell, R. L., et al. 1997, ApJ, 486, 862

Remijan, A. J., Hollis, J. M., Snyder, L. E., Jewell, P. R., \& Lovas, F. J. 2006, ApJ, 643, L37

Solomon, P. M., Jefferts, K. B., Penzias, A. A., \& Wilson, R. B. 1971, ApJ, 168, L107

Solomon, P. M., Penzias, A. A., Jefferts, K. B., \& Wilson, R. B. 1973, ApJ, 185, L63

Snyder, L. E., \& Buhl, D. 1973, Nature Phys. Sci., 243, 45

Urban, Š., Pracna, P., \& Graner, G. 1995, J. Mol. Spectrosc., 169, 185

Verkruijsse, H. D., \& Brandsma, L. 1991, Synth. Comm., 21, 141

Walmsley, C. M., Jewell, P. R., Snyder, L. E., \& Winnewisser, G. 1984, A\&A, 134, L11

Ware, J. M., \& Roberts, J. A. 1984, J. Chem. Phys., 81, 1215

Wlodarczak, G., Bocquet, R., Bauer, A., \& Demaison, J. 1988, J. Mol. Spectrosc., 129, 371 